Anglais de spécialité et milieux professionnels

\title{
Catherine Resche (dir.), La Mise en récit dans les discours spécialisés
}

Berne : Peter Lang, 2016

\section{Jacqueline Percebois}

\section{OpenEdition}

\section{Journals}

Édition électronique

URL : http://journals.openedition.org/asp/4929

DOI : 10.4000/asp.4929

ISSN : 2108-6354

\section{Éditeur}

Groupe d'étude et de recherche en anglais de spécialité

Édition imprimée

Date de publication : 1 mars 2017

Pagination : 173-178

ISSN : 1246-8185

\section{Référence électronique}

Jacqueline Percebois, "Catherine Resche (dir.), La Mise en récit dans les discours spécialisés », ASp [En ligne], 71 | 2017, mis en ligne le 01 mars 2017, consulté le 05 novembre 2020. URL : http:// journals.openedition.org/asp/4929; DOI : https://doi.org/10.4000/asp.4929

Ce document a été généré automatiquement le 5 novembre 2020

Tous droits réservés 


\section{Catherine Resche (dir.), La Mise en récit dans les discours spécialisés}

Berne : Peter Lang, 2016

Jacqueline Percebois

\section{RÉFÉRENCE}

Resche, Catherine (dir.). 2016. La Mise en récit dans les discours spécialisés. Coll.

Aspects linguistiques et culturels en discours spécialisés. Berne : Peter Lang. 261 p. ISBN 978-3-0343-2068-9. 
1 Cet ouvrage collectif, paru chez Peter Lang en 2016 et dirigé par Catherine Resche, est le deuxième numéro de la collection «Aspects linguistiques et culturels des discours spécialisés », également dirigée par Catherine Resche. Il comprend pour l'essentiel des communications présentées aux journées d'étude qu'elle a organisées à l'Université Paris Sorbonne en avril 2015, sur le thème de «La mise en récit dans les discours spécialisés ». L'ouvrage se compose d'un avant-propos, onze articles, une présentation des auteurs, un index des notions et un index des noms propres.

C. Resche commence son avant-propos en contextualisant l'ouvrage dans le cadre des discours spécialisés de communautés

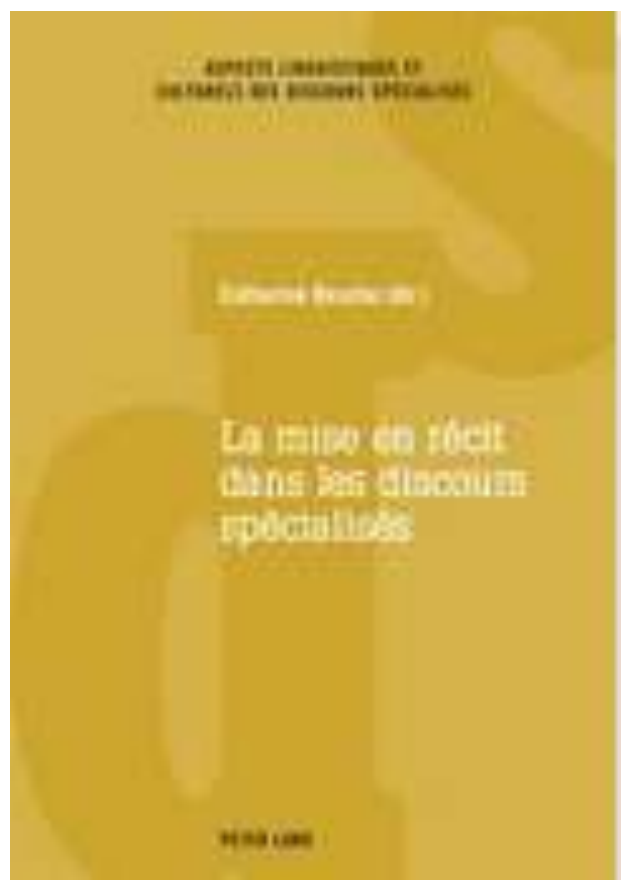
disciplinaires ou professionnelles. Elle rappelle que l'intérêt pour le récit s'est jusqu'ici limité à la littérature et que le projet à l'origine de ses journées d'étude et de l'ouvrage tendait à rechercher son illustration dans d'autres domaines. Elle brosse ensuite un panorama de l'approche traditionnelle $\mathrm{du}$ récit, évoquant les moments narratifs parallèles aux moments explicatifs et descriptifs, soulignant que les domaines spécialisés proposent une plus large gamme de supports que la littérature. Une étape importante de cet avant-propos est la justification du choix du terme " mise en récit », " une affaire de communication entre narrateur et destinataire » (p. xii), et l'exposé des raisons d'éviter la confusion avec le storytelling.

3 L'ouvrage vise à étudier les ressorts de la mise en récit dans des contextes variés. Deux articles ont une perspective historique, sur la marine américaine (Saber) et sur le Watergate (Peynaud). Cinq abordent le domaine scientifique, les découvertes (Rossi), la biologie synthétique (Fries), la recherche expérimentale dans les cahiers de laboratoire (Rowley-Jolivet \& Carter-Thomas), les sciences dures (Le Cor) et les articles de vulgarisation scientifique (Pic \& Furmaniak). Deux se situent dans le monde des entreprises, le site web America's Farmers (Domenec) et Wal-Mart (Resche), enfin les deux derniers ont trait à l'environnement (Biros) et au droit juridictionnel (Charret-Del Bove).

Dans la première contribution, «Mythe, praxéologie, croyances: traits du récit historique au sein de l'US Navy ", Anthony Saber étudie la place de l'histoire dans la marine américaine où il observe une forte présence institutionnelle, le Naval History and Heritage Command et le US Naval Institute exerçant un rôle mémoriel et patrimonial majeur. Il établit une typologie détaillée des textes historiques produits par l'US Navy : récit chronique, mémorialiste, exemplaire, fondateur ou argumentatif. Son choix se porte sur un corpus de cinq ouvrages d'histoire navale - représentatifs de la voix institutionnelle de la marine américaine - dont il souligne le tour anecdotique contribuant à la fonction cohésive du récit. Au fil de son analyse, se dégage le travail de reconstruction des événements par l'historien dont la mise en récit peut varier du 
caractère laudateur des ship histories à la vignette hagiographique, de nombreux récits présentant toutefois une vision plus objective. Par ailleurs, l'auteur relève la dimension praxéologique du récit historique, évaluation des pratiques et parfois rapport d'expert sur leur efficacité. En définitive, il constate que, quel que soit le type de récit, c'est l'histoire d'une marine idéale établie sur des valeurs-clés qu'écrivent les historiens de l'US Navy.

5 Dans la contribution suivante, "The reporters that brought down Nixon: l'affaire du Watergate comme récit constitutif de l'identité professionnelle des journalistes aux États-Unis ", Caroline Peynaud étudie la mise en récit moderne de cet événement. Après avoir examiné la notion de mise en récit et la relation entre récit, mythe et culture professionnelle, elle présente un corpus de dix-sept textes destinés au grand public, publiés en 2012 et en 2014, dates anniversaires du cambriolage et de la démission de Richard Nixon. Si la construction du récit du Watergate - mise en fiction en 1974 dans l'ouvrage de Woodward et Bernstein, All the President's Men, et le film éponyme de Robert Redford sorti en 1976 - était caractérisée par l'héroïsation des deux journalistes et le mystère autour de leur source, le corpus fait apparaître aujourd'hui la déconstruction du récit hérö̈que, critiquant la médiatisation des journalistes et soulignant le rôle déterminant des institutions dans l'enquête, plusieurs articles cherchant à réhabiliter l'image de Nixon. En fin de compte, si le mythe du Watergate apparaît comme le symbole d'une époque révolue, sa remise en récit moderne est l'occasion de revenir aux sources du journalisme d'investigation et de promouvoir les bonnes pratiques professionnelles.

6 Le premier des cinq auteurs s'intéressant au domaine scientifique, Micaela Rossi, met l'accent sur "la nature narrative intrinsèque au texte de démonstration scientifique " (p. 67) dans "Métaphores et mise en récit dans la modélisation des découvertes scientifiques : stratégies de la narration dans l'œuvre de François Jacob ». Son corpus se compose d'écrits du biologiste François Jacob ainsi que des discours de Jacob, Lwoff et Monod lors de la remise de leur Prix Nobel de médecine (1965). Examinant les différences de schéma narratif de la découverte dans les discours des trois chercheurs, elle distingue le schéma habituel de la narration personnelle héroïque et l'ethos de valorisation chez Jacob et Monod de l'éthos de modestie et l'effacement derrière les données objectives chez Lwoff. Parmi les stratégies de mise en récit de la science, elle montre la place essentielle qu'occupent l'analogie et la métaphore, qui n'a plus seulement la fonction ornementale de la tradition rhétorique mais un rôle d'outil heuristique. Et si, pour Jacob, la métaphore est un instrument de structuration dans la formulation d'une théorie, pour Lwoff, elle est limitée à sa fonction didactique. Enfin, chez Monod, c'est un outil rhétorique à la fonction narrative dominante, visant à transformer le récit de la découverte scientifique en roman policier.

7 La métaphore sert également la mise en récit dans l'article de Marie-Hélène Fries, "Métaphores et mise en récit: le cas de la biologie synthétique aux États-Unis », qui porte sur les transcriptions des exposés oraux des séances de la Commission américaine de bioéthique ainsi que sur son rapport final. Ces documents, caractérisés par leur multidisciplinarité, relatent l'expérience scientifique à l'origine de la synthèse du premier génome bactérien entièrement conçu par ordinateur, le rapport final intégrant le récit de cette synthèse dans une mise en récit générale de la biologie synthétique. L'auteur étudie les différences de style de la narration dans les communications orales, où abondent les anecdotes et les références à des récits connus, et dans les rapports 
officiels rédigés collectivement et de façon anonyme. Au-delà des récits visibles exposant les faits, elle montre que des histoires invisibles peuvent être perçues grâce à trois métaphores constitutives de la biologie synthétique : la construction moléculaire, l'ADN comme code linguistique ou informatique et l'usine moléculaire. Ayant mis en évidence les fonctions heuristique et pédagogique de ces métaphores, elle s'efforce d'évaluer leur influence sur le choix des valeurs et les recommandations de la Commission qui place de grands espoirs dans cette science émergente.

C'est un éclairage inédit que portent Elizabeth Rowley-Jolivet et Shirley Carter-Thomas sur le récit de la recherche dans leur article «La vraie histoire de la recherche expérimentale? Comparaison entre la narration de la recherche dans les cahiers de laboratoire et dans les articles de recherche ». Elles y analysent la mise en récit de la recherche construite au jour le jour dans le cahier mis en ligne par un jeune chercheur en bio-informatique à l'université de Boston, dans le cadre du mouvement Open Science, marquant la volonté de partager son récit avec un public plus large que celui des membres de son laboratoire. Les auteurs montrent comment les choix structurels et linguistiques sont liés aux objectifs et au lectorat de chaque genre spécifique. Elles notent ainsi l'exhaustivité des données textuelles et visuelles brutes du récit chronologique du cahier par rapport au caractère synthétique de l'article de recherche. Le recours à trois matrices textuelles de M. Hoey (2001) leur permet de montrer les variations structurelles du cahier, qui contrastent avec les composantes IMRD de l'article de recherche, tandis que l'analyse linguistique des caractéristiques grammaticales met en lumière le caractère très personnel du cahier par rapport à l'impersonnalité de l'article de recherche.

Observant qu'il est communément admis que les seuls récits du domaine scientifique soient historiques, didactiques ou de vulgarisation, Gwen Le Cor s'attache à mettre en évidence la valeur narrative des écrits scientifiques dans l'article «La mise en récit dans les sciences dures : de l'élaboration théorique à la démonstration mathématique ». C'est dans cette perspective qu'elle analyse trois modes de mise en récit dans les sciences dures, dans les expériences de pensée, les objets-images et le raisonnement mathématique. Elle cite en particulier de nombreux exemples d'expériences de pensée de la physique et des mathématiques montrant que la mise en récit, outil cognitif, peut avoir une fonction de modélisation mentale. Quant aux objets-images, elle évoque la valeur narrative des diagrammes dont elle suggère le caractère métaphorique, des raccourcis d'écriture et des démonstrations mathématiques, comme autant de formes de mise en récit. Envisageant le principe et l'utilité d'une pédagogie par la narration pour initier les élèves à la démonstration mathématique, elle fait ainsi apparaitre la fonction didactique de cette mise en récit. Enfin, même si la pensée scientifique n'est pas principalement communicationnelle, G. Le Cor relève le pouvoir des récits dans la transmission d'informations scientifiques au grand public.

10 L'astronomie, l'économie, l'histoire, les mathématiques appliquées et la philosophie sont les cinq disciplines représentées dans le corpus de langue anglaise de l'article d'Elsa Pic et Gregory Furmaniak sur «Formes et fonctions de la narration dans les articles de vulgarisation scientifique en anglais ». À partir des divisions rhétoriques classiques et de la synthèse de deux courants de pensée modernes, les auteurs montrent l'intérêt des modes rhétoriques pour relier genre et grammaire. Leur typologie des modes rhétoriques se fonde sur le plan pragmatique, discursif et formel, tous les modes étant associés à certains traits grammaticaux. Par la description des 
propriétés structurelles et discursives de la narration, les auteurs font ressortir deux grandes classes de fonctions pragmatiques qu'ils qualifient de "non subordonnées, telles que les fonctions informatives ou documentaires» ou de «subordonnées, telles que les fonctions argumentatives ou explicatives» (p. 146). Ils observent des différences d'emploi et donc de fonctions de la narration dans les deux genres dans toutes les disciplines. Les auteurs concluent que le discours scientifique vulgarisé est particulièrement riche sur le plan rhétorique. Toutes les fonctions identifiées du récit s'y retrouvent à des degrés divers, avec une certaine prédilection pour celles qu'ils qualifient de subordonnées.

11 Dans le monde de l'entreprise, l'article de Fanny Domenec intitulé « How technology has changed our lives: la mise en récit du discours de l'entreprise dans America's Farmers ", aborde la mise en récit numérique de ce discours par un site web sponsorisé par Monsanto, le lien avec l'entreprise étant exposé dans la dénomination du site, America's Farmers brought to you by Monsanto, dont le logo figure en bas de chaque page. S'étant intéressée à la communauté de discours à l'origine d'America's Farmers en se demandant quels intérêts sont défendus à travers le site, l'auteur constate la mince frontière séparant les récits individuels, relais indirect du discours de l'entreprise, et le discours promotionnel dès lors que les agriculteurs font référence aux produits de Monsanto. Et si, à partir de 2012, le Farm Blog revêt effectivement une fonction narrative, les récits des expériences individuelles des producteurs prenant le pas sur le discours de l'entreprise, elle s'interroge sur une possible scénarisation d'un discours favorable à l'entreprise, une remise en récit visant à légitimer ses activités. L'auteur conclut que ce site est un support de communication hybride, "à l'intersection des domaines de l'information, de l'argumentation et du divertissement » (p. 180).

12 Au récit tendant à donner une image positive de l'entreprise, Catherine Resche oppose un contre-récit révélateur d'une réalité plus nuancée dans "La mise en récit dans les discours de l'entreprise. Le cas de Wal-Mart». Le corpus comprend des récits internes, autobiographie du fondateur et rapports annuels, incluant faits, anecdotes et rappel des valeurs et, comme récits externes, deux ouvrages sur Wal-Mart et divers autres documents. Elle note que l'autobiographie est en fait un récit polyphonique où coexistent de multiples genres différents. Quant aux rapports annuels, ils illustrent la culture de la légende et filent la métaphore du phare pour une entreprise qui montre la voie. Les deux ouvrages cités comme contre-récit exposent des témoignages de manquements tels que refus de la syndicalisation et impact négatif sur l'environnement auxquels s'ajoutent les pétitions en ligne les dénonçant et les actions en justice rapportées par la presse. La réponse de Wal-Mart au contre-récit, exprimant volonté de transformation et engagements de bonne conduite, figure dans les lettres aux actionnaires et les rapports. En définitive, l'auteur conclut que c'est bien l'histoire de la société de consommation aux États-Unis qui est évoquée à travers la mise en récit de l'entreprise par Wal-Mart et ses détracteurs.

13 Si l'analyse du discours de l'environnement est souvent abordée, le thème choisi par Camille Biros pour illustrer la mise en récit est particulièrement original. En effet, dans son étude, «Récits sur et par les Maoris dans le droit de l'environnement en NouvelleZélande ", C. Biros étudie comment les traditions narratives maories, transmises par des sources orales, ont fait évoluer les lois sur la protection de l'environnement. Elle relate comment l'écoute des narrations des Maoris est devenue une étape essentielle de l'enquête du tribunal de Waitangi, chargé d'examiner les plaintes pour non-respect du 
Traité de Waitangi (1840) qui préservait les droits des Maoris sur leurs territoires. Son analyse du discours du Deed of Settlement, qui suit les négociations et précède le vote d'un texte de loi au Parlement, permet d'apprécier l'importance de la mise en récit des éléments de mythologie, le récit ayant ainsi une fonction d'explication de la vision du monde des Maoris. L'auteur montre notamment le rôle du récit historique dans l'accord sur la protection de la rivière Whanganui. Outre la fonction pédagogique du récit qui cherche à expliquer des concepts, cette étude souligne sa fonction pragmatique dans un contexte de communication interculturelle.

Enfin, c'est le domaine juridique qui sert de cadre à l'étude de Marion Charret-Del Bove sur la " Mise en récit dans les discours juridictionnels de common law anglaise ", où elle examine un corpus composé de cinq décisions de justice relatives à l'application du droit au silence au Royaume-Uni, rendues par la House of Lords entre 1914 et 1993. L'auteur relève plusieurs types de discours, y compris argumentatif et narratif, dans le discours juridictionnel en droit anglais dont elle souligne la polyphonie. Observant que les juges de common law intègrent de nombreux segments narratifs dans leurs décisions de justice, elle définit une fonction narrative et testimoniale du juge dont la mise en récit est une réorganisation des éléments factuels pertinents dans le contexte de l'histoire procédurale qui conduit une affaire devant la plus haute juridiction. Les ultimes mises en récit sont la publication de l'arrêt sur le site du British and Irish Legal Information Institute et dans des revues spécialisées telles que The Weekly Law Reports, dont les éditeurs font précéder le texte de la décision d'un paratexte introductif et explicatif qui constitue une nouvelle recontextualisation de la décision de justice.

Cet ouvrage est remarquable à plus d'un titre : en premier lieu par le fait d'aborder les domaines spécialisés à partir du thème de la mise en récit, traité seulement dans l'ouvrage de M. Gotti et C. Sancho Guinda (2013), en lançant autant de pistes attrayantes de recherche; ensuite par l'intérêt que représente, nous l'avons déjà évoqué, la variété des domaines abordés, et plus encore par la multiplicité des supports pris en compte dans les articles: site internet d'une institution de la marine américaine, ouvrages d'histoire navale (Saber), articles de presse (Peynaud), transcriptions de conférences (Rossi), exposés oraux et rapport de commission gouvernementale (Fries), cahier de laboratoire (Rowley-Jolivet \& Carter-Thomas), articles de recherche scientifique (Rowley-Jolivet \& Carter-Thomas ; Pic \& Furmaniak), article de vulgarisation scientifique (Pic \& Furmaniak), récit numérique (Domenec), autobiographie et rapports annuels d'entreprise (Resche), transcriptions de traditions orales (Biros), arrêts publiant des décisions de justice et revues juridiques (Charret-Del Bove). Par ailleurs, la contribution à l'étude de la métaphore doit être saluée, non seulement dans les articles de M. Rossi et M.-H. Fries, mais également, par petites touches, dans plusieurs autres articles.

Un autre objet spécifique d'intérêt dans l'ouvrage est l'analyse structurelle évoquée par différents auteurs, entre autres les choix structurels dans le cahier de laboratoire et l'article de recherche et les propriétés structurelles avec les matrices textuelles de M. Hoey (Rowley-Jolivet \& Carter-Thomas), l'évocation des étapes du récit canonique (Domenec, Resche, Biros) ou encore la macro-structure de la décision de justice (Charret-Del Bove). Par ailleurs, il convient de ne pas passer sous silence les apports terminologiques de cet ouvrage parmi lesquels : l'exemplum et blue/brown water navy (Saber, pp. 13 et 18); Watergate saga (Peynaud, p. 39) ; junk DNA et gène égoïste (Rossi, p. 59); les amalgames ou mots-valises: Woodstein (Peynaud, p. 33); prosumer et agvocate 
(Domenec, pp. 171 et 174) ou frankenbacteria et biobricks (Fries, pp. 82 et 86), qui ne manqueront pas de retenir l'intérêt des terminologues et pourraient faire l'objet d'un prolongement de cette recherche reliant terminologie et mise en récit.

17 Si chaque article a des qualités spécifiques dans son approche du sujet, son choix de domaine, de corpus et son type d'analyse, l'ouvrage présente une unité quant à l'étude de son objet, la mise en récit des discours spécialisés, par les nombreuses références bibliographiques communes, telles que les ouvrages de Jean-Michel Adam, Roland Barthes ou Gérard Genette, le sérieux des méthodes d'investigation et la clarté des exposés. Il se caractérise aussi par son ouverture à des thèmes et à des genres multiples, et parfois peu ou pas abordés dans la recherche, tels le cahier de laboratoire ou certaines transcriptions de documents oraux. Enfin, l'intérêt culturel que présente chacun des articles recommande la lecture de cet ouvrage bien au-delà de la communauté des chercheurs du domaine de l'anglistique et des spécialistes du discours.

\section{BIBLIOGRAPHIE}

GOTTI, Maurizio \& Carmen SANCHO GUINDA (dir.). 2013. Narratives in Academic and Professional Genres. Berne : Peter Lang.

HOEY, Michael. 2001. Textual Interaction. Londres : Routledge

\section{AUTEURS}

\section{JACQUELINE PERCEBOIS}

Aix-Marseille Université, LERMA EA 853, catherineresche@club-internet.fr 